\title{
Recent Advances in Studies on Structure and Symbiosis-Related Function of Rhizobial K-Antigens and Lipopolysaccharides
}

\author{
Anke Becker, Nicolas Fraysse, and Larissa Sharypova \\ Lehrstuhl für Genetik, Fakultät für Biologie, Universität Bielefeld, Postfach 100131, D-33501 Bielefeld, Germany \\ Submitted 20 April 2005. Accepted 25 May 2005.
}

Exopolysaccharides (EPSs) and K polysaccharides (K-antigens, capsular polysaccharides, or KPSs) are important for the recognition of the symbiotic partner and the infection process, whereas lipopolysaccharides (LPSs) may function at a later stage of symbiosis. Recently, considerable progress has been made in the structural investigation of rhizobial $\mathrm{K}$ antigens and LPSs. This structural data, together with the availability of more and more mutant data, allows new insights into the structure-function relationships of surface polysaccharides and the mode of their action on host cells. This review focuses on rhizobial LPSs and K-antigens. It gives a condensed overview of the recent developments in analysis of their structures and roles during symbiosis.

Additional keyword: lipid A, rhizobium.

Rhizobial surface polysaccharides are of high importance in the establishment of the specific symbiosis between rhizobia and legumes. They play major roles in the recognition of the symbiotic partner and the infection process. The role of exopolysaccharides (EPSs) and capsular polysaccharides (KPSs, $\mathrm{K}$ polysaccharides, or $\mathrm{K}$-antigens) in these early steps has attracted continuous interest (Fraysse et al. 2003; Spaink 2000). An involvement of lipopolysaccharides (LPSs) in the establishment of the Rhizobium spp.-legume symbiosis also was discussed (Kannenberg et al. 1998; Noel and Duelli 2000). Despite extensive research, the exact function of these surface polysaccharides in this process is still unclear. Recent genomic data and progress in analytical techniques, such as mass spectrometry, have facilitated studies of K-antigens and LPS structures. This review gives a condensed overview of new features of the structural elucidation of LPSs and KPSs and their biological impact in symbiosis.

\section{K polysaccharides.}

Rhizobial K polysaccharides show structural analogies to group II K-antigens found in Escherichia coli. The first structures of rhizobial K-antigens were reported for Sinorhizobium fredii USDA205 and $S$. meliloti $\mathrm{Rm} 41$ by Reuhs and associates (1993). Nearly all K-antigens found in rhizobia exhibit a dimeric repeating unit of one hexose, that can be diversely substituted, and a 3-deoxy-D-manno-2-octulosonic acid (Kdo) or a related sugar (Reuhs et al. 1993, 1995). In S. meliloti and

Corresponding author: A. Becker; Telephone: +49 521 106-4824; Fax: +49 521 106-5626; E-mail: Anke.Becker@Genetik.Uni-Bielefeld.DE
S. fredii, antigenic variation of strains is determined by K-antigens, but not by O-antigens of LPSs (Reuhs et al. 1998). It recently has been reported that $S$. meliloti strain Rm1021 produces a low molecular mass $\mathrm{K}$ polysaccharide that is composed exclusively of $\beta$ - $(2 \rightarrow 7)$-linked Kdo residues (Fraysse et al. 2005) (Fig. 1A).

In the recent years, significant progress has been made in the molecular-genetic analysis of the biosynthesis of the $S$. meliloti $\mathrm{Rm} 41 \mathrm{~K}_{\mathrm{R}} 5$ antigen. It consists of disaccharide repeating units composed of glucuronic and pseudaminic acids, which are decorated by butyryl and acetyl residues (Reuhs et al. 1993, 1998) (Fig. 1B). Three gene clusters are involved in the production of this K-antigen (Kereszt et al. 1998; Putnoky et al. 1990) (Fig. 2). The chromosomal $r k p-1$ (former fix-23) region seems to determine the biosynthesis of a specific lipid carrier that is required for KPS synthesis (Kiss et al. 1997; Petrovics et al. 1993). The chromosomal $r k p-2$ region (lpsL, $r k p K$ ) is involved in metabolism of nucleotide diphosphosugars. Both genes in this region are required for LPS production, whereas only $r k p K$ is required for the synthesis of KPS (Kereszt et al. 1998). The $r k p-3$ gene region located on the pSymB megaplasmid includes 10 genes. Six of these genes, rkpLMNOPQ, are involved in synthesis of strain-specific sugar precursors and determine the saccharide composition of the $\mathrm{K}_{\mathrm{R}} 5$ K-antigen (Kiss et al. 2001). S. meliloti Rm1021 also contains $r k p-1$ and $r k p-2$ gene regions, but there are only partial similarities to the $r k p-3$ gene cluster in the Rm1021 genome (Kiss et al. 2001). It contains genes similar to $r k p R, r k p S, r k p T$, and $r k p Z$ that may be involved in export and polymerization of KPS. There are no homologous counterparts to the biosynthesis genes $r k p L M N O P Q$. Three putative paralogs of the $r k p Z$ gene of Rm41 are present in this region of the Rm1021 genome, but only one of these seems to encode a full-length protein. Previously, an $r k p Z$ (lpsZ) gene homolog could not be detected by Southern hybridizations in Rm1021 (Williams et al. 1990).

In the light of recent findings, the $\operatorname{rkp} A B C D E F$ genes from the $r k p-1$ deserve special attention. When they first were identified in Rm41, these six genes were annotated as genes encoding a fatty acid synthase (FAS) (Petrovics et al. 1993). Moreover, the biochemical function of $\mathrm{RkpF}$ as an acyl carrier protein was demonstrated (Epple et al. 1998). However, currently available data allows a better prediction of their function. $r k p A B C D E F$ encoded proteins resemble domains of a multifunctional type I polyketide synthase (PKS). Therefore, it is not a surprise that, in $\mathrm{Rm} 1021$, the $\operatorname{rkp} A B C D E F$ genes are fused into a single open reading frame, named $r k p A$, which encodes a long polypeptide of 2,504 amino acids. Type I polyketide synthases are 
multifunctional proteins exerting a number of sequential enzymatic reactions. Lipid biosynthesis via PKS is fundamentally different from the FAS pathway. Although PKSs use the same basic reactions as the FAS pathway, the cycle of fatty acid condensation and elongation often is abbreviated, so that a highly derivatized carbon chain is produced, typically containing many keto and hydroxy groups as well as carbon-carbon double bonds (Wallis et al. 2002).

Until now, the enterobacterial paradigm of KPS biosynthesis has dominated. Therefore, it seemed puzzling that $S$. meliloti employed a PKS-derived lipid for production of K polysaccharides. Based on the analysis of prokaryotic genome sequences, it has become clear that recruitment of PKS-derived lipids in K polysaccharide production is not a unique feature of $S$. meliloti. An ortholog of S. meliloti $1021 \mathrm{rkpA}$ was identified in Burkholderia mallei (DeShazer et al. 2001) and Bordetella bronchiseptica (Parkhill et al. 2003). In both organisms, the orthologous gene, named $w c b R$, is situated within KPS gene clusters (DeShazer et al. 2001; Parkhill et al. 2003).

Since identification of the $\operatorname{rkp} A B C D E F$ genes in $S$. meliloti Rm41 (Petrovics et al. 1993), it was assumed that a lipid carrier or anchor is required for the biosynthesis of $\mathrm{K}$ polysaccharide. However, no lipid moiety was detected in high molecular mass K-antigen of Rm41 (Reuhs et al. 1993). Recent investigation of low molecular mass $\mathrm{K}$ polysaccharide of strain Rm1021 revealed a lipid anchor attached to the polyKdo saccharide (Fraysse et al. 2005). Preliminary analyses indicated that this lipid probably includes phosphoglycerol and a long acyl chain. It will be of interest to elucidate the relationship between this lipid anchor and the predicted PKS-derived polyketide.

\section{Role of K polysaccharides in symbiosis.}

In enteric bacteria, capsular polysaccharides form a hydrated matrix that contributes to protection against abiotic factors (Roberts 1996). In rhizobia, K polysaccharides may have the same function. In addition, they may protect against legume defense products during the infection process. It also is possible that rhizobial $\mathrm{K}$-antigens play an active role during symbiosis. Evidence for the active, probably signaling, role of $\mathrm{K}$ polysaccharides first was gained from a $S$. meliloti $\mathrm{Rm} 41$ exoB mutant. This mutant is unable to synthesize EPSs but successfully infects the alfalfa host plant (Reuhs et al. 1995; Williams et al. 1990), indicating that $\mathrm{K}$ polysaccharide from this strain can substitute for EPS. A rkpZ mutation in Rm41 affected the size distribution of K-polysaccharide and led to the loss of symbiotic activity (Reuhs et al. 1995; Williams et al. 1990).

High molecular mass K polysaccharide, whose size exceeded the resolution capacity of polyacrylamide gel electrophoresis (PAGE), was identified in Rm1021 (Reuhs et al. 1995). This K polysaccharide, in contrast to that of Rm41, was symbiotically inactive. The difference in size and activity of $\mathrm{K}$ polysaccharides from Rm1021 and Rm41 was attributed to the rkpZ gene (Reuhs et al. 1995; Williams et al. 1990). Introduction of the $r k p Z$ gene of $\mathrm{Rm} 41\left(r k p Z_{\mathrm{Rm} 41}\right)$ into a $\mathrm{Rm} 1021$ exoB mutant that is deficient in production of both EPSs (succinoglycan and
A $\rightarrow 7)-\beta-$ Kdo- $(2 \rightarrow$
with lipid anchor

\section{B $-\beta$-GlcA $\rightarrow$ Pse5Nac7N( $\beta$-OH-But $)-$}

with minor O-acetylation

Fig. 1. Structures of repeating units of $\mathrm{K}$ polysaccharides. A, $\mathrm{K}$ polysaccharide of Sinorhizobium meliloti Rm1021 is a homopolymer of 3deoxy-D-manno-2-octulosonic acid (Kdo) (Fraysse et al. 2005). B, $\mathrm{K}_{\mathrm{R}} 5$ antigen of $S$. meliloti AK631 consists of disaccharide repeating units (Reuhs et al. 1993, 1998; Kiss et al. 2001). Abbreviations: GlcA, Glucuronic acid; Pse, 5,7-diamino-3,5,7,9-tetradeoxynonulosonic acid (pseudaminic acid), Ac, acetyl group; $\beta$-OH-But, $\beta$-OH-butyryl group. galactoglucan) resulted in partial restoration of the symbiotic activity. This strain synthesized $\mathrm{K}$ polysaccharide that could be detected in PAGE (Reuhs et al. 1995). It remains to be resolved whether this $\mathrm{K}$ polysaccharide is derived from the low molecular mass KPS described by Fraysse and associates (2005) by an increase in size or from the high molecular mass KPSs reported by Reuhs and associates (1995) by a size reduction. Whether or not this K polysaccharide, like the low molecular mass KPS (Fraysse et al. 2005), also is composed exclusively of $\beta$ $(2 \rightarrow 7)$-linked Kdo residues remains to be investigated.

The Rm41 exoB mutant (AK631) induced normal nitrogenfixing $\left(\mathrm{Fix}^{+}\right)$nodules on roots of alfalfa, whereas the Rm1021 exoB mutant carrying $r k p Z_{\mathrm{Rm} 41}$ induced more Fix than $\mathrm{Fix}^{+}$ nodules (Reuhs et al. 1995; Williams et al. 1990). This might be due to the strain-specific background, different structural properties, or differences in size distribution of the $\mathrm{K}$ polysaccharides. It is possible that $\mathrm{K}$ polysaccharides from both strains contribute to passive protection, whereas they may differ in their function as signal molecules. The active role of $\mathrm{K}$ antigen seems to affect the promotion of infection thread initiation and development (Pellock et al. 2000; Putnoky et al. 1990; Reuhs et al. 1993).

The symbiotically active fraction of $\mathrm{K}_{\mathrm{R}} 5$ antigen from Rm41 seems to have the same size as the size of symbiotically active EPSI or EPSII (Reuhs et al. 1995). Up to now, it remains unclear how these structurally different oligosaccharides can replace each other during symbiosis (Pellock et al. 2000). A detailed analysis of $\mathrm{K}$ polysaccharide modification caused by expression of the heterologous rkpZ gene in S. meliloti 1021 may provide new clues to the structural requirements of bacterial oligosaccharides for promotion of nodule infection.

\section{LPSs.}

LPSs are anchored in the outer membrane. Structures and biosynthesis of rhizobial LPSs were reviewed extensively by Carlson and associates (1999), Price (1999), and Noel and Duelli (2000). LPS molecules consist of a lipid A anchor that is associated with a core polysaccharide. This core polysaccharide can be substituted by an $\mathrm{O}$-antigen polysaccharide moiety. The low molecular weight form of LPS (rough LPS) contains only the lipid A and core domains, whereas all three associated domains constitute the high molecular weight form of LPS (smooth LPS or O-antigen LPS). LPS core oligosaccharides were isolated after mild acid hydrolysis and gel filtration or high-performance anion-exchange chromatography purification, and partially or fully characterized in Rhizobium leguminosarum bv. phaseoli (Bhat et al. 1991, 1994; Carlson et al. 1989), R. trifolii ANU843 (Carlson et al. 1988), R. etli (Forsberg and Carlson 1998), S. fredii, different S. meliloti strains, and Sinorhizobium sp. NGR 234 (Reuhs et al. 1998).

Considerable progress recently has been made in the structural analysis of rhizobial lipid As. In general, they consist of a 2,3-diamino glucose or a glucosamine disaccharide unit, 6'linked to the core portion through a Kdo (Fig. 3). Mainly four to six $\alpha$-hydroxy fatty acids are carried by the glucosaminodisaccharide. In certain cases, the first position is oxidized to amino gluconate or phosphorylated. The nonreducing glucosamine also can be phosphorylated or substituted by a galacturonate moiety in $4^{\prime}$ position. A characteristic feature of rhizobial lipid As is that they all possess a very long-chain fatty acid, mainly $27-\mathrm{OH}-\mathrm{C} 28$ fatty acid, as an acyloxyacyl substituent in $3^{\prime}$ position.

$R$. etli CE3 and $R$. leguminosarum 3855 produce identical lipid As. In both organisms, lipid A consists of a mixture of structurally related species that lack phosphate. The nonreducing glucosamine carries a galacturonic acid residue at $4^{\prime}$ position. The reducing glucosamine can be oxidized to 2-amino-2- 
deoxy-gluconate. The number of acyl substitutions varies from four to five (Que et al. 2000) (Fig. 3B).

Mesorhizobium huakuii lipid A differs from the preceding description (Choma and Sowinski 2004). Here, a di-diaminodideoxyglucose backbone is tetra-, penta-, or hexa-acylated and one phosphate is only partially present in $4^{\prime}$ position. A galacturonate moiety has been found in position 1 (Fig. 3C).

S. meliloti lipid A has been an object of investigations employing genetic and mass spectrometric analyses (Ferguson et al. 2005; Kanipes et al. 2003; Sharypova et al. 2003). Its diglucosamine backbone is bi-phosphorylated and tetra- or pentaacylated (Fig. 3A). The 27-OH-C28 fatty acid is substituted by either a metoxy butyrate or a more commonly observed hydroxy-butyrate, which, for instance, was observed in the lipid A from Sinorhizobium sp. NGR234 (Gudlavalleti and Forsberg 2003). The LPS of this strain, which is closely related to $S$. meliloti, was investigated in detail and a comparable structure with a high degree of $\mathrm{N}$-linked fatty acid length heterogeneity and bi-phosphorylation was found.

The lipid A of Sinorhizobium Sin1 also has been analyzed. The main particularity of its structure is that the glucosamine backbone is devoid of phosphate or uronic acid substituents (Jeyaretnam et al. 2002). Similarly to lipid A of $R$. etli CE3, it is tetra- or penta-acylated, and the proximal glucosamine is oxidized to a gluconate.

The lipid A structure of Agrobacterium tumefaciens, a plantpathogenic bacterium, was completely resolved, and strong structural analogies to the general lipid A structure of rhizobia were found: a biphosphorylated diglucosamine backbone, a similar acylation, pattern and the presence of a very long-chain fatty acid (Silipo et al. 2004).

\section{Role of LPSs in symbiosis.}

LPSs may play various roles at different stages of symbiosis. Possible roles of LPSs during initial recognition, infection initiation, root tissue invasion, bacterial release into plant cells, formation of symbiosomes (compartments containing nitrogenfixing rhizobia surrounded by the plant-derived peribacteroid membrane), and their senescence are discussed by Kannenberg and associates (1998) and Noel and Duelli (2000). In this review, we focus on the recent findings.

LPS is an integral part of the outer membrane of gram-negative bacteria. However, LPS may be released from the cell in the form of vesicles (Beveridge 1999). Therefore, LPSs may act not only during the intimate contact with plant cells but at distance as well.

Although it was demonstrated that purified rhizobial LPS binds rapidly to root hair tips and promotes infection of white clover by $R$. leguminosarum bv. trifolii (Dazzo et al. 1991), it is believed that LPS is not specifically involved in the initial steps of symbiosis, because LPS mutants are able to elicit nodules and at least partially invade root tissue (Spaink 2000). However, it should be noted that LPS mutants used to study the Rhizobium spp.-legume interaction in the 1990s were affected only in the saccharidic portion of LPS. Recently, rhizobial mutants affected in the lipid A part of LPS were constructed. This was accomplished by the inactivation of the $a c p X L$ gene, encoding an acyl carrier protein for the very longchain 27-OH-C28:0 fatty acid in $S$. meliloti and $R$. leguminosarum (Sharypova et al. 2003; Vedam et al. 2003) and by inactivation of the lpxXL gene encoding a lipid A acyltransferase involved in the transfer of the long fatty acid to the lipid A precursor in S. meliloti (Ferguson et al. 2005).

MALDI-TOF-MS analysis of the purified lipid A showed that acpXL or lpxXL mutations resulted in the total disappearance of this fatty acid in $S$. meliloti and $R$. leguminosarum LPS (Ferguson et al. 2005; Sharypova et al. 2003; Vedam et al.
2003). All mutants showed a delayed infection. It was hypothesized that the presence of a very long-chain fatty acid could stabilize the bacterial membrane, and this could support bacterial adaptation inside the nodule.

Recently, it has been demonstrated that LPSs of S. meliloti suppressed an elicitor-induced defense response in a Medicago sativa cell suspension culture (Albus et al. 2001). Addition of yeast elicitor to this culture caused a typical defense reaction, detected by the elevation of hydrogen peroxide concentration and alkalinization of the medium. Addition of either LPS or lipid A, or lipid A lacking the very long acyl chain, inhibited these reactions in a concentration-dependent manner (Scheidle et al. 2005). To exert this activity, lipid A seems to require a structural motif that is shared by all rhizobia. The acylation pattern could be such a prerequisite. All characterized rhizobial lipid As are tetra- or penta-acylated, 3-OH-C14:0 fatty acids are present in 3 and $3^{\prime}$ positions, length heterogeneity is observed for $\mathrm{N}$-linked fatty acids, and it ranges from $\mathrm{C} 14$ to C19. A perception of lipid A seems to be nonspecific and, hence, not involved in host range determination. Moreover, this nonspecific perception may be similar during early symbiotic and pathogenic interactions. Thus, A. tumefaciens lipid A also may help this pathogen to escape early defense responses on a wide range of host plants (Silipo et al. 2004). However, it remains to be elucidated whether cells of the intact plant may respond to the bacterial partner in the same way as was demonstrated for the cultured cells exposed to the "foreign" yeast elicitor and purified rhizobial LPS or lipid A.

A second level of LPS perception by the host plant may occur later, during infection thread growth and endocytosis by the plant cells. At these later stages, bacteria may be exposed to the late host defense reactions, and the host plant may require a new signal for progression of endosymbiosis. Although only few lipid A mutants have been studied so far, at this stage, the structure of the saccharidic part of LPS (core or O-antigen) seems to be more important than that of lipid A. The most severe symbiotic phenotypes were reported for LPS mutants, which are symbionts of plants forming determinate nodules (Kannenberg et al. 1998) R. etli mutant CE166 displaying reduced abundance of $\mathrm{O}$-antigen and lacking a particular sugar residue induced the formation of nonfixing pseudonodules on Phaseolus vulgaris. The mutant was arrested at the stage of nodule penetration (Noel et al. 2000). Sesbania rostrata displayed symptoms of defense reactions against mutants of Azorhizobium caulinodans producing altered LPS. The bacteria accumulated in enlarged infection threads, and only ineffective nodule-like structures were observed (Gao et al. 2001). A similar observation was made for $S$. meliloti lps $B$ mutant Rm6369 producing core-truncated LPS forms. This mutant failed to colonize $M$. truncatula indeterminate nodules efficiently (Niehaus and Becker 1998).

The mode of action of rhizobial LPSs during symbiosis can be diverse. They may act as inhibitors of defense response, signals, a physical barrier, or antigens mimicking a plant-like interface. Inside the nodule, rhizobial LPSs may help bacteria to adapt to the endosymbiotic environment. Physicochemical studies of LPS isolated from $R$. etli strain CE3, which was cultivated under conditions imitating an endosymbiotic environment, low $\mathrm{pH}$, and reduced oxygen concentration, showed that LPS become more hydrophobic (Kannenberg and Carlson 2001). Moreover, cultivation of this strain in the presence of host exudates also resulted in increased hydrophobicity of LPS due to the increased level of hydrophobic 2- $O$-methyl fucose in the O-antigen region (Noel et al. 2004). It was found that addition of host plant flavonoids to Sinorhizobium sp. NGR234 induced the production of a rhamnose-rich LPS, which was normally absent in free-living culture and only 
Rm41 chromosomal rkp-1 (fix-23) region

$r k p$

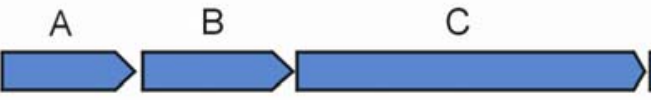

$D \quad E \quad F$
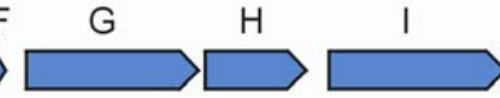

J

Rm1021 chromosomal rkp-1 (fix-23) region

$r k p$

A
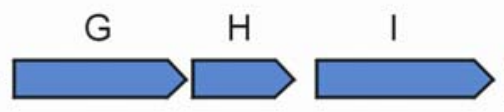

$\mathrm{J}$

\section{Rm41 chromosomal rkp-2 region}

$\stackrel{\text { IpsL }}{\longrightarrow} \mathrm{rkpK}^{\mathrm{r}}$

\section{Rm1021 chromosomal rkp-2 region}

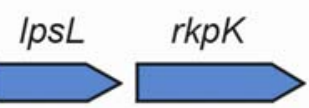

\section{Rm41 rkp-3 region on $p S y m B$}

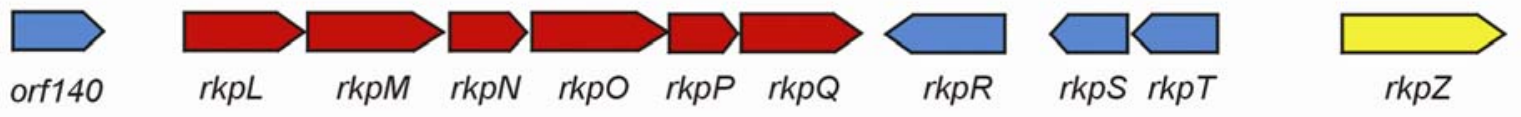

\section{Rm1021 cluster 2 on pSymB}
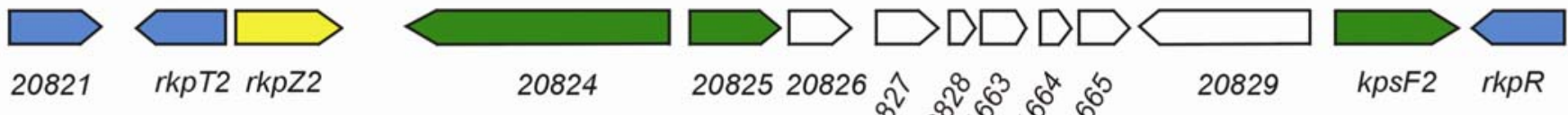

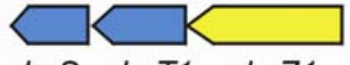

rkpS rkpT1 rkpZ1

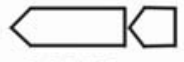

20835

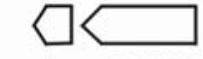

ก 20838

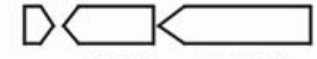

के 2084020841
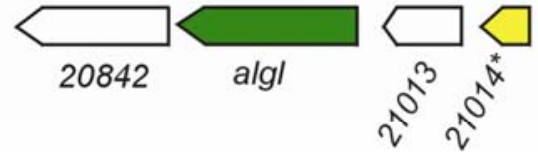

$1 \mathrm{~kb}$ genes related to polysaccharide biosynthesis or transport

$r k p Z$ and its paralogs in 1021 strain-specific KPS biosynthesis genes

genes probably related to polysaccharide biosynthesis

genes probably not related to polysaccharide biosynthesis

Fig. 2. Organization of the $r k p$ gene clusters of Rm41 and related clusters in Rm1021 (S. meliloti 1021 genome project website); $*$ indicates gene with partial homologies to $r k p Z_{\mathrm{Rm} 41}$. 


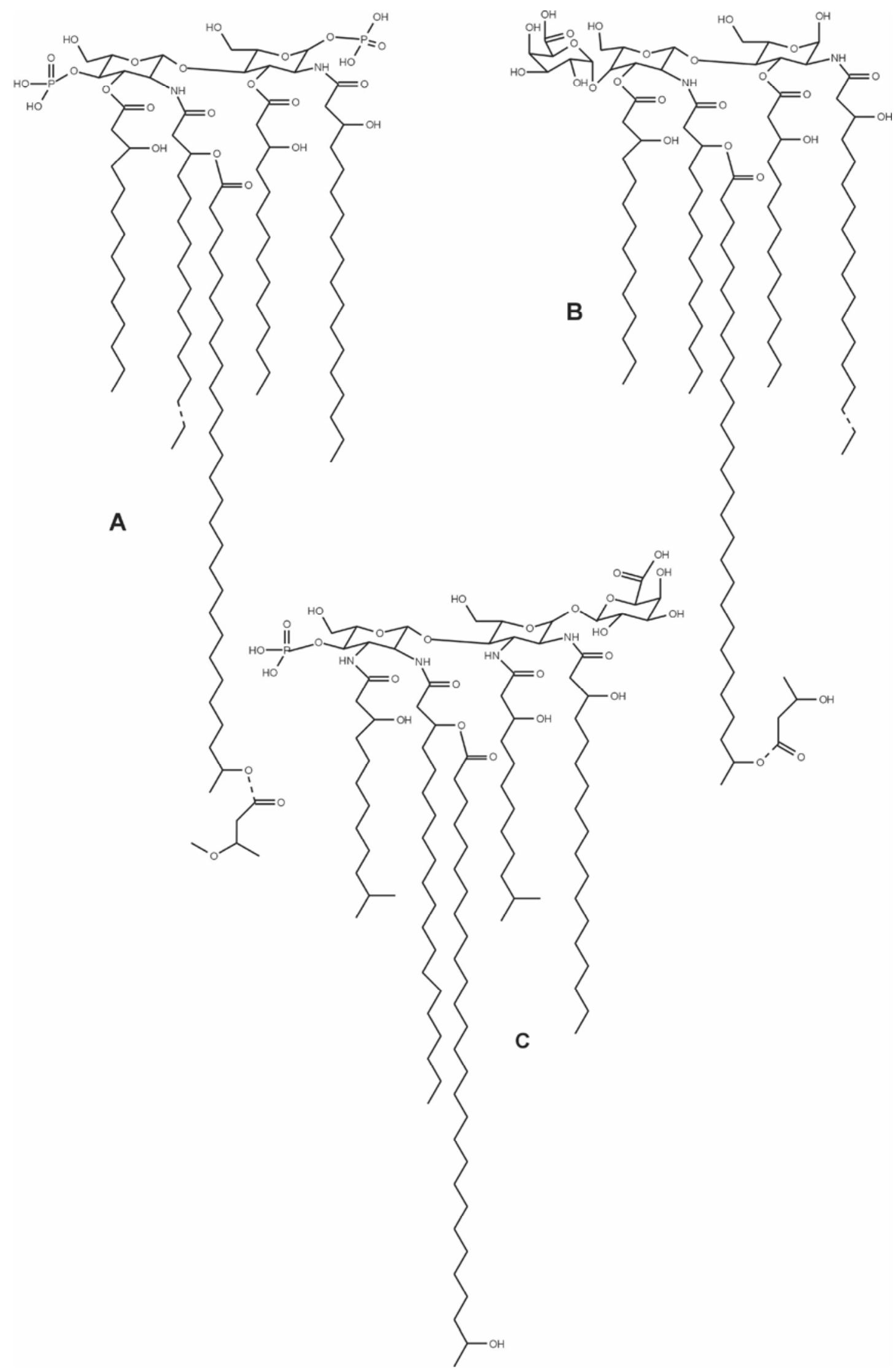

Fig. 3. Examples of different rhizobial lipid A structures that have been recently proposed. For each organism, only one of several lipid A species is presented. A, Sinorhizobium meliloti Rm1021 (Sharypova et al. 2003) and Sinorhizobium sp. strain NGR234 (Gudlavalleti and Forsberg 2003) have similar structures. B, Rhizobium etli lipid A (Que et al. 2000): a galacturonate moiety is observed in 4' position. C, 2,3-diamino-2,3-dideoxyglucose constitutes the saccharidic backbone of Mesorhizobium huakuii lipid A. 3-OH-C13 fatty acids are observed. Dashed lines reflect heterogeneity with different lengths of fatty acids or the nonsystematic presence of 4-hydroxy or methoxy butyrate observed in one strain. 
present inside nodules of Vigna unguiculata (Fraysse et al. 2002). Compared with the LPS expressed by free-living cells of Sinorhizobium sp. NGR234, this rhamnose-rich LPS is characterized by an increased hydrophobicity (Fraysse et al. 2002). An adaptation toward increased hydrophobicity probably begins prior to nodule infection. A comparison of LPS extracted from endosymbiotic and free-living bacteria of other rhizobial species will be required to gain new insights into the mechanisms of such an adaptation.

Recently, Mathis and associates (2005) obtained evidence that LPS of $A$. caulinodans can function as a communication signal. This was shown by characterization of an oac 2 mutant of strain ORS571 producing modified LPS with a reduced rhamnose content and higher electrophoretic mobility compared with normal LPS (Gao et al. 2001). During symbiosis with the host plant Sesbania rostrata, this mutant was arrested at the stage of intercellular multiplication and was unable to fix nitrogen (Mathis et al. 2005). The symbiotic deficiency of this mutant could be restored by co-inoculation with a Nod-factordeficient mutant that produced normal LPS or could be partially restored by the addition of purified LPS. To our knowledge, this is the first report on functional extracellular symbiotic complementation of an LPS mutant. Up to now, such experiments succeeded only with EPS-deficient mutants (Niehaus and Becker 1998). It should be noted that the A. caulinodans$S$. rostrata symbiosis is characterized by specific features not found in other Rhizobium spp.-legume interactions. S. rostrata is a semiaquatic tropical legume that forms nodules on stems and roots. Under hydroponic conditions, azorhizobia invade the host plant via crack entry at the bases of lateral roots or at the bases of adventitious root primordia. In typical Rhizobium spp--legume interactions, bacteria invade the host plant via infection threads growing inside root hairs. Moreover, the $A$. caulinodans LPS may be rather different from rhizobial LPS, because a correlation between LPS structure and phylogeny of bacteria frequently was found (Kannenberg et al. 1998). Therefore, it remains to be elucidated whether results obtained with this $A$. caulinodans mutant are conditioned by specific features of the Azorhizobium-Sesbania symbiotic system or whether extrapolation to other Rhizobium spp.-legume symbioses is possible.

Recent advances in new technologies have opened broad avenues for the study of symbiosis-related LPS functions. Determination of LPS structures and analysis of its biological activities in appropriate model systems will help to unravel the structure-function relationship of these complex macromolecules. If LPS is indeed a signal molecule, the next challenge will be to identify plant receptors. Such studies may be exemplified by the analysis of Nod factor perception during initiation of nodule development (D'Haeze and Holsters 2002) or perception of $E$. coli lipid A during an innate immunity response in animal cells (Raetz and Whitfield 2002). In the latter system, lipid A is recognized specifically by certain host proteins including the signaling receptor TLR-4 (Raetz and Whitfield 2002).

\section{ACKNOWLEDGMENTS}

Work in our laboratory was supported by grant 031U213D from Bundesministerium für Bildung und Forschung, Germany, grant BIZ 7 from Deutsche Forschungsgemeinschaft, and grant HPRN-CT-2002-00251 from the European Community. We thank Karsten Niehaus and Véréna Poinsot for critical discussions.

\section{LITERATURE CITED}

Albus, U., Baier, R., Holst, O., Pühler, A., and Niehaus K. 2001 Suppression of an elicitor-induced oxidative burst in Medicago sativa cell-cul- tures by Sinorhizobium meliloti lipopolysaccharides. New Phytol. 151:597-606

Beveridge, T. J. 1999. Structures of gram-negative cell walls and their derived membrane vesicles. J. Bacteriol. 181:4725-4733.

Bhat, U. R., Forsberg, S. L., and Carlson, R. W. 1994. Structure of lipid A component of Rhizobium leguminosarum bv. Phaseoli lipopolysaccharide. J. Biol. Chem. 269:14402-14410.

Bhat, U. R., Krishnaiah, B. S., and Carlson, R. W. 1991 Re-examination of the structures of the lipopolysaccharides core oligosaccharides from Rhizobium leguminosarum biovar phaseoli. Carbohydr. Res. 220:219227.

Carlson, R. W., Garci, F., Noel, D., and Hollingsworth, R. 1989 The structures of the LPS core components from Rhizobium leguminosarum biovar phaseoli and two of its symbiotic mutants, CE 109, and CE 309. Carbohydr. Res. 195: 101-110.

Carlson, R. W., Hollingsworth, R. L., and Dazzo, F. B. 1988 A core oligosaccharide component from the lipopolysaccharide of Rhizobium trifolii ANU843. Carbohydr. Res. 176:127-135.

Carlson, R. W., Reuhs, B. L., Forsberg, L. S., and Kannenberg, E. L. 1999 Rhizobial cell surface carbohydrates: their structures, biosynthesis, and functions. Pages 53-90 in: Genetics of Bacterial Polysaccharides. J. B. Goldberg, ed. CRC Press, Boca Raton, FL, U.S.A.

Choma, A., and Sowinski, P. 2004 Characterization of Mesorhizobium huakuii lipid A containing both D-galacturonic acid and phosphate residues. Eur. J. Biochem. 271:1310-1322.

Dazzo, F. B., Truchet, G. L., Hollingsworth, R. I., Hrabak, E. M. Pankratz, H. S., Philip-Hollingsworth, S., Salzwedel, J. L., Chapman, K., Appenzeller, L., Squartini, A., Gerhold, D., and Orgameide, G. 1991. Rhizobium lipopolysaccharide modulates infection thread development in white clover root hairs. J. Bacteriol. 173:5371-5384.

DeShazer, D., Waag, D. M., Fritz, D. L., and Woods, D. E. 2001. Identification of a Burkholderia mallei polysaccharide gene cluster by subtractive hybridization and demonstration that the encoded capsule is an essential virulence determinant. Microb. Pathog. 30:253-269.

D'Haeze, W., and Holsters, M. 2002. Nod factor structures, responses, and perception during initiation of nodule development. Glycobiology 12:79R-105R.

Epple, G, van der Drift, K. M., Thomas-Oates, J. E., and Geiger, O. 1998. Characterization of a novel acyl carrier protein, $\mathrm{RkpF}$, encoded by an operon involved in capsular polysaccharide biosynthesis in Sinorhizobium meliloti. J. Bacteriol. 180:4950-4954.

Ferguson, G. P., Datta, A., Carlson, R. W., and Walker, G. C. 2005. Importance of unusually modified lipid A in Sinorhizobium meliloti and legume symbiosis. Mol. Microbiol. 56:68-80.

Forsberg, L. S., and Carlson, R. W. 1998 The structures of the lipopolysaccharides from Rhizobium etli strains CE358 and CE359-The complete structure of the core region of $R$-etli lipopolysaccharides. J. Biol. Chem. 273:2747-2757.

Fraysse, N., Jabbouri, S., Treilhou, M., Couderc, F., and Poinsot, V. 2002 Symbiotic conditions induce structural modifications of Sinorhizobium sp. NGR234 surface polysaccharides. Glycobiology 12:741-748.

Fraysse, N., Couderc, C., and Poinsot, V. 2003 Surface polysaccharides involvement in establishing the Rhizobium-legume symbiosis. Eur. J. Biochem. 270:1365-1380.

Fraysse, N., Lindner, B., Kaczynski, Z., Sharypova, L., Holst, O., Niehaus, K., and Poinsot, V. 2005. Sinorhizobium meliloti strain 1021 produces a low-molecular weight capsular polysaccharide that is a Kdo homopolymer harboring a phospholipidic anchor. Glycobiology 15:101-108.

Gao, M., D’Haeze, W., De Rycke, R., Wolucka, B., and Holsters, M. 2001 Knockout of an Azorhizobial dTDP-L-Rhamnose synthase affects lipopolysaccharide and extracellular polysaccharide production and disables symbiosis with Sesbania rostrata. Mol. Plant-Microbe Interact. 14:857-866.

Gudlavalleti, S. K., and Forsberg, L. S. 2003 Structural characterization of the lipid A component of Sinorhizobium sp. NGR234 rough and smooth form lipopolysaccharide. Demonstrating that the distal amide-linked acyloxyacyl residue containing the long chain fatty acid is conserved in Rhizobium and Sinorhizobium sp. NGR234. J. Biol. Chem. 278:39573968.

Jeyaretnam, B., Glushka, J., Kolli, V. S., and Carlson, R. W. 2002 Characterization of a novel lipid-A from Rhizobium species Sin-1. A unique lipid-A structure that is devoid of phosphate and has a glycosyl backbone consisting of glucosamine and 2-aminogluconic acid. J. Biol. Chem. 277:41802-41810.

Kanipes, M. I., Kalb, S. R., Cotter, R. J., Hozbor, D. F., Lagares, A., and Raetz, R. H. 2003 Relaxed sugar donor selectivity of a Sinorhizobium meliloti ortholog of the Rhizobium leguminosarum mannosyl transferase $L p c C$. J. Biol. Chem. 278:16365-16371.

Kannenberg, E. L., and Carlson, R. W. 2001 Lipid A and O-chain modifi- 
cations cause Rhizobium lipopolysaccharides to become hydrophobic during bacteroid development. Mol. Microbiol. 39:379-391.

Kannenberg, E. L., Reuhs, B. L. Forsberg, L. S., and Carlson, R. W. 1998. Lipopolysaccharides and $\mathrm{K}$ antigens: their structures, biosynthesis and functions. Pages 119-154 in: The Rhizobiaceae: Molecular Biology of Model Plant-Associated Bacteria. H. Spaink, A. Kondorosi, and P. Hooykaas, eds. Kluwer Academic Publishers, Dordrecht, The Netherlands.

Kereszt, A., Kiss, E., Reuhs, B. L., Carlson, R. W., Kondorosi, A., and Putnoky, P. 1998. Novel rkp gene clusters of Sinorhizobium meliloti involved in capsular polysaccharide production and invasion of the symbiotic nodule: the $r k p K$ gene encodes a UDP-glucose dehydrogenase. J. Bacteriol. 180:5426-5431.

Kiss, E., Reuhs, B. L., Kim, J. S. Kereszt, A., Petrovics, G., Putnoky, P., Dusha, I., Carlson R. W., and Kondorosi, A. 1997. The rkpGHI and -J genes are involved in capsular polysaccharide production by Rhizobium meliloti. J. Bacteriol. 179:2132-2140.

Kiss, E., Kereszt, A., Barta, F., Stephens, S., Reuhs, B. L., Kondorosi, A. and Putnoky, P. 2001 The rkp-3 gene region of Sinorhizobium meliloti Rm41 contains strain-specific genes that determine $\mathrm{K}$ antigen structure. Mol. Plant-Microbe Interact. 14:1395-1403.

Mathis, R., Van Gijsegem, F., De Rycke, R., D'Haeze, W., Van Maelsaeke, E., Anthonio, E., Van Montagu, M. Holsters, M., and Vereecke, D. 2005 Lipopolysaccharides as a communication signal for progression of legume endosymbiosis. Proc. Natl. Acad. Sci. U.S.A. 102:2655-2660.

Niehaus, K., and Becker, A. 1998 The role of microbial surface polysaccharides in the Rhizobium-legume interaction. Subcell. Biochem. 29:73-116.

Noel, K. D., and Duelli, D. M. 2000 Rhizobium lipopolysaccharide and its role in symbiosis. Pages 415-431 in: Prokaryotic Nitrogen Fixation: A Model System for Analysis of Biological Process. E. W. Triplett, ed. Horizon Scientific Press, Wymondham, UK.

Noel, K. D., Forsberg, L. S., and Carlson, R. W. 2000 Varying the abundance of $\mathrm{O}$ antigen in Rhizobium etli and its effect on symbiosis with Phaseolus vulgaris. J. Bacteriol. 182:5317-5324.

Noel, K. D., Box, J. M., and Bonne, V. J. 2004 2-O-methylation of fucosyl residues of a rhizobial lipopolysaccharide is increased in response to host exudate and is eliminated in a symbiotically defective mutant. Appl. Environ. Microbiol. 70:1537-1544.

Parkhill, J., Sebaihia, M., Preston, A., Murphy, L. D., Thomson, N., Harris, D. E., Holden, M. T., Churcher, C. M., Bentley, S. D., Mungall, K. L. Cerdeno-Tarraga, A. M., Temple, L., James, K., Harris, B., Quail, M. A., Achtman, M., Atkin, R., Baker, S., Basham, D., Bason, N., Cherevach, I., Chillingworth, T., Collins, M., Cronin, A., Davis, P., Doggett, J., Feltwell, T., Goble, A., Hamlin, N., Hauser, H., Holroyd, S., Jagels, K., Leather, S., Moule, S., Norberczak, H., O'Neil, S., Ormond, D., Price, C., Rabbinowitsch, E., Rutter, S., Sanders, M. Saunders, D., Seeger, K., Sharp, S., Simmonds, M., Skelton, J., Squares, R., Squares, S., Stevens, K., Unwin, L., Whitehead, S., Barrell, B. G., and Maskell, D. J. 2003. Comparative analysis of the genome sequences of Bordetella pertussis, Bordetella parapertussis and Bordetella bronchiseptica. Nat. Genet. 35:32-40.

Pellock, B. J., H. P. Cheng, and Walker, G. C. 2000 Alfalfa root nodule invasion efficiency is dependent on Sinorhizobium meliloti polysaccharides. J Bacteriol. 182:4310-4318.

Petrovics, G., Putnoky, P., Reuhs, B. Kim, J., Thorp, T. A., Noel, K. D., Carlson, R. W., and Kondorosi, A. 1993. The presence of a novel type of surface polysaccharide in Rhizobium meliloti requires a new fatty acid synthase-like gene cluster involved in symbiotic nodule development. Mol. Microbiol. 8:1083-1094.

Price, N. P. J. 1999 Carbohydrate determinants of Rhizobium-legume symbioses. Carbohydr. Res. 317:1-9.

Putnoky, P., Petrovics, G., Kereszt, A., Grosskopf, E., Ha, D. T., Banfalvi, Z., and Kondorosi, A. 1990 Rhizobium meliloti lipopolysaccharide and exopolysaccharide can have the same function in the plant-bacterium interaction. J. Bacteriol. 172:5450-5458.

Que, N. L. S., Ribeiro, A. A., and Raetz, C. R. H. 2000 Two-dimensional NMR spectroscopy and structures of six Lipid A species from Rhizobium etli CE3. J. Biol. Chem. 275:28017-28027.

Raetz, C. R., and Whitfield, C. 2002. Lipopolysaccharide endotoxins Annu. Rev. Biochem. 71:635-700.

Reuhs, B. L., Carlson, R. W., and Kim, J. S. 1993 Rhizobium fredii and Rhizobium meliloti produce 3-deoxy-D-manno-2-octulosonicacid-containing polysaccharides that are structurally analogous to group II K antigens (capsular polysaccharides) found in Escherichia coli. J. Bacteriol. 175:3570-3580.

Reuhs, B. L., Geller, D. P., Kim, J. S., Fox, J. E., Kolli, V. S., and Pueppke, S. G. 1998 Sinorhizobium fredii and Sinorhizobium meliloti produce structurally conserved lipopolysaccharides and strain-specific K antigens. Appl. Environ. Microbiol. 64:4930-4938.

Reuhs, B. L., Williams, M. N. V., Kim, J. S., Carlson, R. W., and Cote, F. 1995. Suppression of the FixZ phenotype of Rhizobium meliloti exoB mutants by lps $Z$ is correlated to a modified expression of the $\mathrm{K}$ polysaccharide. J. Bacteriol. 177:4289-4296.

Roberts, I. S. 1996. The biochemistry and genetics of capsular polysaccharide production in bacteria. Annu. Rev. Microbiol. 50:285-315.

Scheidle, H., Gross, A., and Niehaus, K. 2005 The Lipid A substructure of the Sinorhizobium meliloti lipopolysaccharides is sufficient to suppress the oxidative burst in host plants. New Phytol. 165:559-565.

Sharypova, L. A., Niehaus, K., Scheidle, H., Holst, O., and Becker, A. 2003 Sinorhizobium meliloti AcpXL mutant lacks the C28 hydroxylated fatty acid moiety of lipid A and does not express a slow migrating form of lipopolysaccharide. J. Biol. Chem. 278:12946-12954.

Silipo, A., De Castro, C., Lanzetta, R., Molinaro, A., and Parrilli, M. 2004 Full structural characterization of the lipid A components from the $\mathrm{Ag}$ robacterium tumefaciens strain C58 lipopolysaccharide fraction. Glycobiology 14:805-815

Spaink, H. P. 2000. Root nodulation and infection factors produced by rhizobial bacteria. Annu. Rev. Microbiol. 54:257-288.

Vedam, V., Kannenberg, E. L., Haynes, J. G., Sherrier, D. J., Datta, A., and Carlson R. W. 2003 A Rhizobium leguminosarum acpXL mutant produces lipopolysaccharide lacking 27-hydroxyoctacosanoic acid. J. Bacteriol. 185:1841-1850.

Wallis, J. G., Watts, J. L., and Browse, J. 2002. Polyunsaturated fatty acid synthesis: what will they think of next? Trends Biochem. Sci.. 27:467473.

Williams, M. N., Hollingsworth, R. I., Klein, S., and Signer, E. R. 1990 The symbiotic defect of Rhizobium meliloti exopolysaccharide mutants is suppressed by $l p s Z^{+}$, a gene involved in lipopolysaccharide biosynthesis. J. Bacteriol. 172:2622-2632.

\section{AUTHOR-RECOMMENDED INTERNET RESOURCE}

Sinorhizobium meliloti 1021 genome project websites: bioinfo.genopoletoulouse.prd.fr/annotation/iANT/bacteria/rhime www.cebitec.uni-bielefeld.de/groups/nwt/sinogate 